Timo Tigges*, Ludwig Wiegank, Lorik Bajrami, Alexandru-Gabriel Pielmuş, Michael Klum, Aarne Feldheiser, and Reinhold Orglmeister

\title{
Heart Rate Variability Analysis During Lower Body Negative Pressure Test Induced Central Hypovolemia
}

https://doi.org/10.1515/cdbme-2019-0017

\begin{abstract}
In clinical patient monitoring scenarios, the detection of hemorrhage is still a major problem. Traditional vital signs like heart rate and blood pressure are insensitive to blood loss due to compensatory mechanisms in the body that can sustain these parameters until shortly before cardiovascular collapse. These compensatory mechanisms during blood loss are primarily driven by the autonomic nervous system. Heart rate variability analysis is a viable tool in the quantitative analysis of the autonomic nervous system and shows promising results in the context of hypovolemia detection. In order to investigate if HRV parameters suitably reflect a mild to moderate blood volume reduction, we conducted a lower body negative pressure test study with 30 volunteering participants thereby simulating progressive central hypovolemia. Here, HRV parameters from the time domain (mean HR, SDNN, RMSSD, rSDRM, pNN50), the frequency domain (VLF, LF, HF, LF/HF), non-linear HRV parameters (SD1, SD2, SD1/SD2, SampEn, ApEn) and the respiratory rate (RR) were collected. The changes of the evaluated parameters as a consequence of the reduced blood volume were statistically evaluated. A statistically significant deviation from their baseline values could be found for RMSSD, rSDRM, pNN50, HF, LF/HF, SD1 and SD1/SD2 at a chamber pressures starting at $-30 \mathrm{mmHg}$. Therefore, we support the proposition that heart rate variability analysis can help in detecting otherwise occult hypovolemia.
\end{abstract}

Keywords: Hypovolemia Detection, Heart Rate Variability, Respiration, Lower Body Negative Pressure Test

\footnotetext{
*Corresponding author: Timo Tigges, Lorik Bajrami, Alexandru-Gabriel Pielmuş, Michael Klum, Reinhold Orglmeister, Chair of Electronics and Medical Signal Processing, Technische Universität Berlin, Einsteinufer 17, Berlin, Germany, e-mail: timo.tigges@tu-berlin.de

Ludwig Wiegank, Department of Anaesthesiology and Operative Intensive Care Medicine, Campus Charité Mitte and Campus Virchow-Klinikum, Charité - University Medicine Berlin, Berlin, Germany

Aarne Feldheiser, Klinik für Anästhesiologie und Intensivmedizin, Universitätsklinikum Essen und Universität Duisburg-Essen, Essen, Germany
}

\section{Introduction}

Undetected hemorrhage is still a major problem in clinical patient monitoring. Traditional vital signs like heart rate and blood pressure are insensitive to blood loss due to compensatory mechanisms in the body that can sustain these parameters until shortly before cardiovascular collapse [7]. Therefore, it is desirable to investigate novel cardiovascular parameters that are sensitive even to mild levels of blood loss in order to allow for an early detection of otherwise occult hemorrhage. Heart Rate Variability (HRV) is a viable tool in the quantitative analysis of the autonomic nervous system (ANS). As compensatory mechanisms during hypovolemia are primarily driven by the ANS [8], HRV analysis could give further insight into the patient status that is not revealed by standard hemodynamic monitoring equipment like blood pressure and oxygen saturation monitors.

Previous studies already showed promising results when using HRV parameters for the detection of impeding cardiovascular collapse ([2, 5]). In this work, we investigate if HRV parameters could also be used to indicate a mild to moderate blood volume loss. For this reason, we conducted a lower body negative pressure (LNBP) test study to simulate a blood volume reduction in-vivo and retrospectively analysed the reaction of HRV parameters from the time and the frequency domain, of non-linear HRV parameters and of the respiration.

\section{Material and methods}

\subsection{LBNP study}

We conducted the LBNP test study with 30 male healthy volunteers aging between 21 and 35 years. We collected informed consent from all participants prior to the start of the test. After being familiarized with the laboratory equipment, the volunteers were asked to lie down in a supine position with their lower body placed in the LBNP chamber.

Progressive central hypovolemia was induced by stepwise increment of LBNP from $0 \mathrm{mmHg}$ (baseline) over 
$-15 \mathrm{mmHg}$ (mild blood loss, $\approx 10 \%$ of total blood volume) and $-30 \mathrm{mmHg}$ (moderate blood loss, $\approx 10-20 \%$ of total blood volume) to $-45 \mathrm{mmHg}$ (severe blood loss, $>20 \%$ of total blood volume [3]), where each LBNP stage was sustained for $7 \mathrm{~min}$. Subsequently, the LBNP was gradually reduced until reaching $0 \mathrm{mmHg}$ again, followed by a $10 \mathrm{~min}$ recovery period that was also recorded. The study protocol was carried out in accordance with the Declaration of Helsinki. Registration of the study was performed internationally [4] and ethical vote was attained from the ethical commission (Ethics Committee of Charité - University Medicine Berlin, No. EA1/249/17).

Data from the same study has also been used for the evaluation of pulse arrival and pulse transit times. Corresponding results are presented in [12].

During the study protocol, we recorded the ECG (Einthoven I) from the thorax with a micro-controller based measurement system as it is presented in [11]. The ECG signal was recorded with a sampling rate of 500 samples/s. Individual R-peaks were automatically detected. Subsequently, a manual review of the annotations was conducted, where faulty detections were corrected and ectopic beats were excluded from any further analysis.

\subsection{HRV parameter estimation}

In order to evaluate the development of HRV in the conducted LBNP study, HRV parameters in different domains were calculated. In the time domain, the following HRV parameters were calculated: the standard deviation of normal-to-normal beat intervals (SDNN), the root-mean square of successive normal-to-normal beat interval differences (RMSSD), the ratio between the total variabiliy the short time variability (rSDRM) and the fraction of adjacent normal-to-normal beats whose lengths differ by more than $50 \mathrm{~ms}$ (pNN50) [6].

In the frequency domain, the power in the very low frequency range $(\mathrm{VLF},<0.04 \mathrm{~Hz})$, the power in the low frequency range $(\mathrm{LF}, 0.04 \mathrm{~Hz}-0.15 \mathrm{~Hz})$ and the power in the high frequency range $(\mathrm{HF}, 0.15 \mathrm{~Hz}-0.4 \mathrm{~Hz})$ were calculated. Additionally, the parameter LF/HF was calculated as the ratio between LF and HF [6]. Power spectral density estimation has been conducted with the Lomb-Scargle method.

Regarding the non-linear HRV parameters, two HRV parameters were calculated from the return map (i.e. the Poincaré plot) of successive normal-to-normal intervals: the standard deviation along the identity axis of the return map (SD1) and the standard deviation along the perpendicular axis (SD2) [9]. Additionally, the sample entropy (SampEn) and the approximate entropy (AppEn) were calculated [10].

\subsection{Respiration rate estimation}

For the evaluation of respiratory changes as a consequence of the simulated blood volume reduction induced by the LBNP test, the respiration rate (RR) was estimated from the recorded ECG signal. For this purpose, the RRest toolbox was utilized [1] as follows: First, the amplitude and time values of the detected R-peaks and the corresponding Q-points were used to derive a baseline wander (BW), an amplitude modulation (AM) and a frequency modulation (FM) signal from the ECG. These three signals were then fused by averaging and the resulting signal was interpolated to a uniform sampling rate of 5 samples/s. Next, the respiration rate was extracted by windowing the interpolated signal into time intervals with a length of $32 \mathrm{~s}$, transforming each window into the frequency domain and identifying the dominant frequency component in the frequency interval between $0.67 \mathrm{~Hz}$ and $0.5 \mathrm{~Hz}$ (i.e. 4 to 30 breaths per minute).

\subsection{Statistical evaluation}

The HRV parameters were estimated based on the last $5 \mathrm{~min}$ of every LBNP pressure level. This evaluation interval length corresponds to the suggested length of short-term HRV analysis [6]. A single value per LNBP pressure level was also calculated for the electrically derived respiration rate by taking the median of all RR measurements in the same 5 min intervals.

In order to evaluate if the distribution of the calculated HRV and respiration parameters recorded during the LBNP pressure stages are significantly different to the their corresponding values of the baseline measurements, the paired Wilcoxon signed-rank test was conducted for each recorded pair of HRV parameters and LBNP phases. Additionally, relative changes of each parameter with respect to its corresponding baseline value were calculated in order to investigate subject specific trends in the data. The testing for statistical significance was conducted for a p-value of 0.001 .

\section{Results and Discussion}

We successfully completed the entire $40 \mathrm{~min}$ protocol in 28 of the subjects. The results of the estimated HRV parameters are presented separately for the time domain HRV measures (Table 1), the frequency domain HRV measures (Table 2), the nonlinear HRV measures (Table 3 ) and the respiratory rate (Table 4).

Regarding the time domain HRV measures, it can be seen that HR is slightly slowed down at an LBNP pressure level of $-15 \mathrm{mmHg}$ with regards to its baseline values. At higher 
Tab. 1: Development of time domain HRV measures during LBNP test. All values are given as median (inter-quantile range). Statistically significant $(p<0.001)$ deviations from the baseline are marked by $(\cdot)^{\dagger}$.

\begin{tabular}{lccccc}
\hline \hline & Baseline & $-15 \mathrm{mmHg}$ & $-30 \mathrm{mmHg}$ & $-45 \mathrm{mmHg}$ & \multicolumn{1}{c}{ Recovery } \\
\hline HR (bpm) & $71.6(13.7)$ & $69.9(14.0)$ & $72.8(14.3)$ & $78.9(15.5)^{\dagger}$ & $67.4(12.9)^{\dagger}$ \\
$\mathcal{R}_{\text {Baseline }}(\%)$ & & $-2.2(3.3)$ & $4.6(7.9)$ & $11.9(14.9)$ & $-6.3(6.7)$ \\
RMSSD (ms) & \multirow{2}{*}{$31.5(29.5)$} & $32.7(20.0)$ & $24.1(16.3)^{\dagger}$ & $18.8(7.2)^{\dagger}$ & $34.9(24.2)$ \\
$\mathcal{R}_{\text {Baseline (\%) }}$ & & $-9.1(34.2)$ & $-25.2(20.8)$ & $-35.6(20.0)$ & $22.8(42.9)$ \\
SDNN (ms) & $50.0(21.3)$ & $52.2(17.3)$ & $48.4(16.7)$ & $42.4(15.5)$ & $58.0(23.4)^{\dagger}$ \\
$\mathcal{R}_{\text {Baseline (\%) }}$ & & $-4.4(27.9)$ & $-10.0(24.1)$ & $-11.4(24.3)$ & $19.8(41.9)$ \\
rSDRM (1) & \multirow{2}{*}{$0.5(0.6)$} & $1.6(0.5)$ & $1.9(0.6)^{\dagger}$ & $2.3(0.8)^{\dagger}$ & $1.5(0.6)$ \\
$\mathcal{R}_{\text {Baseline (\%) }}$ & & $1.2(16.4)$ & $25.8(36.4)$ & $38.3(46.8)$ & $1.4(20.1)$ \\
pNN50 (\%) & \multirow{2}{*}{$7.5(26.4)$} & $9.6(20.3)$ & $3.3(12.0)^{\dagger}$ & $1.5(3.1)^{\dagger}$ & $13.2(23.5)$ \\
$\mathcal{R}_{\text {Baseline }}(\%)$ & & $-11.2(59.3)$ & $-54.6(51.4)$ & $-79.2(27.4)$ & $54.6(151.8)$ \\
\hline \hline
\end{tabular}

LBNP pressures, the HR shows an increase that is found statistically significant from its baseline values only at $-45 \mathrm{mmHg}$. Starting at $-30 \mathrm{mmHg}$, RMSDD and pNN50 are signficantly decreased, whereas rSDRM is the significantly increased.

The frequency domain analysis mainly demonstrates a reduction in cardiac variability in the HF band. The median spectral energy in the RF band is gradually reduced from a median of $485.1 \mathrm{~ms}^{2}$ during the baseline measurements down to $189.9 \mathrm{~ms}^{2}$ at $-45 \mathrm{mmHg} \mathrm{LBNP}$, corresponding to a median reduction of $57.4 \%$. In contrast, VLF and LF are not significantly altered by the conducted hemorraghe simulation. Therefore, it can be reasoned that the changes in the $\mathrm{LF} / \mathrm{HF}$ ratio is mainly caused by the HF component.

Of the non-linear HRV measures, only SD1 showed significant differences to its baseline. At $-30 \mathrm{mmHg}$ it is reduced by a median of $25.2 \%$ and at $-45 \mathrm{mmHg}$ it is reduced by a median of $35.6 \%$.

In median, the respiratory rate estimated from an electrically derived respiration signal is gradually increase with increasing simulated blood loss, although statistical significance could not be established for the given significance level.

\section{Conclusion}

We investigated the effect of a simulated progressive blood loss on HRV parameters from the time and frequency domain, on the non-linear HRV parameters and on the respiration. It could be established that a part of the evaluated HRV parameters show a significant reaction to a moderate blood volume loss. During a mild blood volume reduction, no statistical significance could be established. Thus, for an early hemorraghe detection, it appears advisable to combine the HRV-based hypovolemia monitoring with other cardiovascular parameters like pulse arrival times or pulse waveform parameters. We are currently focusing our research effort on this sensor fusion approach.

\section{Author Statement}

The author state no funding involved. Authors state no conflict of interest. Informed consent has been obtained from all individuals included in this study. The research related to human use complies with all the relevant national regulations, institutional policies and was performed in accordance with the tenets of the Helsinki Declaration, and has been approved by the authors' institutional review board or equivalent committee.

\section{References}

[1] P. H. Charlton, D. A. Birrenkott, T. Bonnici, M. A. F. Pimentel, A. E. W. Johnson, J. Alastruey, L. Tarassenko, P. J. Watkinson, R. Beale, and D. A. Clifton. Breathing Rate Estimation From the Electrocardiogram and Photoplethysmogram: A Review. IEEE Reviews in Biomedical Engineering, 11:2-20, 2018.

[2] W. H. Cooke, C. A. Rickards, K. L. Ryan, and V. A. Convertino. Autonomic compensation to simulated hemorrhage monitored with heart period variability. Critical Care Medicine, 36(6):1892-1899, jun 2008.

[3] W. H. Cooke, K. L. Ryan, and V. A. Convertino. Lower body negative pressure as a model to study progression to acute hemorrhagic shock in humans. Journal of Applied Physiology, 96(4):1249-1261, 2004. PMID: 15016789.

[4] A. Feldheiser. Plethysmographic Pulse-Contour and PulseWave-Transit-Times For Haemodynamic Evaluation In Bleeding Simulation (Plethysmo). ClinicalTrials.gov, 2018. ClinicalTrials.gov Identifier: NCT03481855. Accessed: Jan. 28, 2019.

[5] S.-Y. Ji, A. Belle, K. R. Ward, K. L. Ryan, C. A. Rickards, V. A. Convertino, and K. Najarian. Heart rate variability analysis during central hypovolemia using wavelet transformation. 
Tab. 2: Development of frequency domain HRV measures during LBNP test. All values are given as median (inter-quantile range). Statistically significant $(p<0.001)$ deviations from the baseline are marked by $(\cdot)^{\dagger}$.

\begin{tabular}{|c|c|c|c|c|c|}
\hline & Baseline & $-15 \mathrm{mmHg}$ & $-30 \mathrm{mmHg}$ & $-45 \mathrm{mmHg}$ & Recovery \\
\hline $\operatorname{VLF}\left(\mathrm{ms}^{2}\right)$ & $890.2(742.9)$ & $819.8(621.9)$ & $906.7(608.7)$ & $725.5(690.7)$ & $1378.3(1685.5)$ \\
\hline $\mathcal{R}_{\text {Baseline }}(\%)$ & & $-10.1(60.6)$ & $4.5(71.7)$ & $-9.1(83.7)$ & $42.2(137.1)$ \\
\hline $\mathrm{LF}\left(\mathrm{ms}^{2}\right)$ & $832.8(495.8)$ & $740.5(484.2)$ & $647.4(571.0)$ & $614.2(336.6)$ & $847.4(796.2)$ \\
\hline $\mathcal{R}_{\text {Baseline }}(\%)$ & & $-3.9(45.4)$ & $-14.6(57.4)$ & $-25.7(41.3)$ & $29.3(\quad 71.9)$ \\
\hline $\mathrm{HF}\left(\mathrm{ms}^{2}\right)$ & $485.1(876.5)$ & $425.2(351.0)$ & $285.2(315.2)^{\dagger}$ & $189.9(177.7)^{\dagger}$ & $569.1(851.4)$ \\
\hline $\mathcal{R}_{\text {Baseline }}(\%)$ & & $-14.4(45.6)$ & $-40.6(40.6)$ & $-57.4(33.0)$ & $42.6(142.7)$ \\
\hline $\mathrm{LF} / \mathrm{HF}(1)$ & $1.8(1.8)$ & $2.1(\quad 2.2)$ & $2.4(2.5)^{\dagger}$ & $3.6(4.2)^{\dagger}$ & $1.5(\quad 1.4)$ \\
\hline $\mathcal{R}_{\text {Baseline }}(\%)$ & & $5.4(60.6)$ & $56.2(82.4)$ & $80.3(182.5)$ & $-19.1(48.2)$ \\
\hline
\end{tabular}

Tab. 3: Development of non-linear HRV measures during LBNP test. All values are given as median (inter-quantile range). Statistically significant $(p<0.001)$ deviations from the baseline are marked by $(\cdot)^{\dagger}$.

\begin{tabular}{|c|c|c|c|c|c|}
\hline & Baseline & $-15 \mathrm{mmHg}$ & $-30 \mathrm{mmHg}$ & $-45 \mathrm{mmHg}$ & Recovery \\
\hline SD1 (ms) & $22.3(20.9)$ & $23.2(14.1)$ & $17.1(11.5)^{\dagger}$ & $13.3(5.1)^{\dagger}$ & $24.7(17.1)$ \\
\hline $\mathcal{R}_{\text {Baseline }}(\%)$ & & $-9.1(34.2)$ & $-25.2(20.8)$ & $-35.6(20.0)$ & $22.8(42.9)$ \\
\hline SD2 (ms) & $67.2(24.4)$ & $69.9(22.5)$ & $66.7(21.8)$ & $58.1(21.1)$ & $78.8(0.2)^{\dagger}$ \\
\hline $\mathcal{R}_{\text {Baseline }}(\%)$ & & $-3.2(25.8)$ & $-5.1(23.8)$ & $-7.6(26.0)$ & $19.1(35.8)$ \\
\hline SD1/SD2 (\%) & $36.8(15.5)$ & $33.2(12.7)$ & $27.2(9.8)^{\dagger}$ & $22.7(8.8)^{\dagger}$ & $35.0(13.6)$ \\
\hline $\mathcal{R}_{\text {Baseline }}(\%)$ & & $-0.8(17.4)$ & $-22.1(27.1)$ & $-31.3(27.3)$ & $-1.7(22.7)$ \\
\hline SampEn (1) & $1.3(0.6)$ & $1.1(0.3)$ & $1.2(0.3)$ & $1.0(0.3)$ & $1.3(0.5)$ \\
\hline $\mathcal{R}_{\text {Baseline }}(\%)$ & & $0.2(46.9)$ & $-10.8(34.1)$ & $-21.1(49.2)$ & $3.2(59.2)$ \\
\hline ApEn (1) & $1.1(0.2)$ & $1.1(0.3)$ & $1.1(0.2)$ & $1.0(0.2)$ & $1.3(0.2)^{\dagger}$ \\
\hline $\mathcal{R}_{\text {Baseline }}(\%)$ & & $1.3(16.6)$ & $-3.3(16.6)$ & $-5.4(24.5)$ & $12.1(23.7)$ \\
\hline
\end{tabular}

Tab. 4: Development of respiratory rate during LBNP test. All values are given as median (inter-quantile range). Statistically significant $(p<0.001)$ deviations from the baseline are marked by $(\cdot)^{\dagger}$.

\begin{tabular}{lcrccc}
\hline \hline & Baseline & $-15 \mathrm{mmHg}$ & $-30 \mathrm{mmHg}$ & $-45 \mathrm{mmHg}$ & Recovery \\
\hline $\mathrm{RR}(\mathrm{bpm})$ & $16.2(4.2)$ & $16.3(3.7)$ & $18.1(4.6)$ & $18.6(4.0)$ & $17.2(4.4)$ \\
$\mathcal{R}_{\text {Baseline }}(\%)$ & & $1.7(18.0)$ & $7.4(19.0)$ & $17.1(23.0)$ & $1.4(19.4)$ \\
\hline \hline
\end{tabular}

Journal of Clinical Monitoring and Computing, 27(3):289302, feb 2013.

[6] M. Malik, A. J. Camm, J. T. Bigger, G. Breithardt, S. Cerutti, R. J. Cohen, and D. H. Singer. Heart rate variability. Standards of measurement, physiological interpretation, and clinical use. European Heart Journal, 17(3):354-381, 1996.

[7] S. McGee, W. B. Abernethy III, and D. L. Simel. Is This Patient Hypovolemic? JAMA: The Journal of the American Medical Association, 281(11):1022-1029, 031999.

[8] M. Orlinsky, W. Shoemaker, E. D. Reis, and M. D. Kerstein. Current controversies in shock and resuscitation. Surgical Clinics of North America, 81(6):1217-1262, dec 2001.

[9] S. L. Raetz, C. A. Richard, A. Garfinkel, and R. M. Harper. Dynamic Characteristics of Cardiac R-R Intervals during Sleep and Waking States. Sleep, 14(6):526-533, nov 1991.

[10] J. S. Richman and J. R. Moorman. Physiological time-series analysis using approximate entropy and sample entropy.
American Journal of Physiology-Heart and Circulatory Physiology, 278(6):H2039-H2049, jun 2000.

[11] T. Tigges, T. Büchler, A. Pielmuş, M. Klum, A. Feldheiser, O. Hunsicker, and R. Orglmeister. Assessment of In-ear Photoplethysmography as a Surrogate for Electrocardiography in Heart Rate Variability Analysis. In World Congress on Medical Physics and Biomedical Engineering 2018, pages 293-297, Singapore, 2019. Springer Singapore.

[12] T. Tigges, A. Feldheiser, A. Pielmuş, M. Klum, L. Wiegank, and R. Orglmeister. Evaluation of Pulse Arrival Times during Lower Body Negative Pressure Test for the Non-Invasive Detection of Hypovolemia. In 2019 41th Annual International Conference of the IEEE Engineering in Medicine and Biology Society (EMBC). IEEE, 2019. 